Reprod. Nutr. Dévelop., 1987, 27 (1 B), 311-312.

\title{
Variations de composition et d'activité LPL des tissus adipeux abdominaux selon le lieu de prélèvement chez la chèvre en fin de gestation
}

P. BAS, F. GALLOUIN, P. MORAND-FEHR, Annie ROUZEAU, J. HERVIEU

Station de nutrition et alimentation II.N.R.A.I de I'I.N.A.-PG, 16, rue Claude-Bernard, 75231 Paris Cedex 05.

Summary. The lipid content, LPL activity and fatty acid composition were studied at several sites in the omental (OM), perirenal (PR) and mesenteric (MES) adipose tissues of five pregnant goats. PR seemed to be the most homogeneous tissue and OM the least. Two thin OM sites seemed to have lower lipid content, lower C18:0 and higher $18: 1, n-9$ percentages than the others. LPL activity seemed to be higher in one site near the rumen.

Chez les porcs et les bovins, un gradient de composition et d'activités enzymatiques a été mis en évidence dans le tissu adipeux sous-cutané en relation avec la proximité de la peau $(1,5)$ mais peu d'études ont été effectuées sur des tissus adipeux internes (3). Aussi nous avons voulu étudier les variations de la composition à l'intérieur de 3 tissus adipeux afin de déterminer la représentativité des échantillons par rapport au tissu total.

Matériel et méthodes. La teneur en lipides (2), l'activité de la LPL (4) et la composition en acides gras (AG) sont déterminés dans différents échantillons, repérés anatomiquement dans les tissus omental $(\mathrm{OM})$, périrénal $(P R)$ et mésentérique (MES) de 5 chèvres adultes en $19^{\circ}$ semaine de gestation. Onze échantillons ont été prélevés au centre et à la périphérie du tissu OM (fig. 1). Le $11^{\mathrm{e}}$ est proche $d^{\prime} u n$ vaisseau sanguin et les $4^{\theta}$ et $9^{\mathrm{e}}$ proviennent de zones de faible épaisseur. Trois échantillons ont été prélevés dans le tissu PR : le 1er sur la grande courbure rénale, le $2^{\mathrm{e}}$ à $9 \mathrm{~cm}$ du bord postérieur du rein, et le $3^{\mathrm{e}}$ à $18 \mathrm{~cm}$ dans la partie caudale. Trois autres dans le tissu MES : le $1^{\text {er }}$ dans la région iléale, le $2^{\mathrm{e}}$ dans la région duodénale et le $3^{\mathrm{e}}$ au milieu du jejunum.

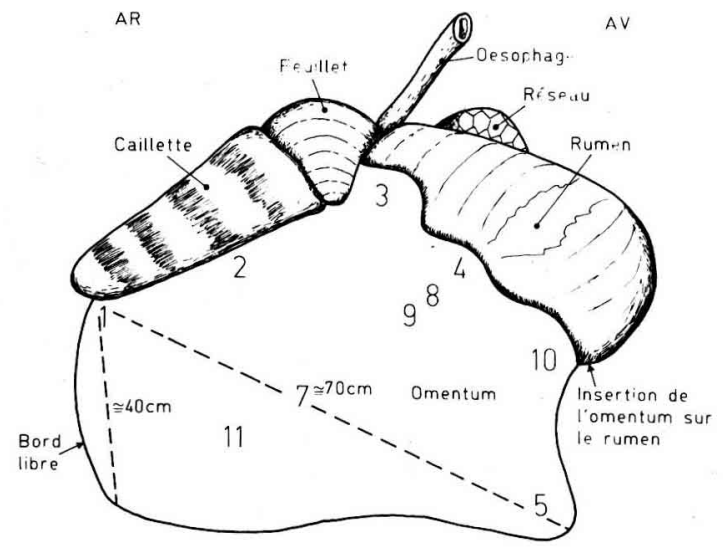

FIG. 1. - Lieux de prélèvement dans le tissu omental de la chèvre. Vue face droite.

Résultats et discussion. Dans le tissu $O M$, les 11 échantillons présentent des teneurs en lipides et des pourcentages d'acides gras différents. Les écarts sont de faible amplitude pour les teneurs en lipides ; cependant ces teneurs sont plus fai- 
bles dans les échantillons $n^{\circ} 4,9$ et 11. Par contre, la différence d'activité LPL est importante entre les échantillons. Ainsi l'activité est nettement plus élevée dans le $n^{\circ} 4$, puis dans une moindre mesure dans les $n^{\circ} 8,9$ et 10 . De même, le $18: 0$ et le $18: 1, n-9$ ont des pourcentages très variables entre les 11 échantillons. Les $n^{\circ} 4$ et 9 sont les plus riches en $18: 1, n-9$ et les plus pauvres en $18: 0$. Le $16: 0$ est plus stable (tabl. 1). Le 18: 0 est corrélé négativement au $18: 1, n-9$ $(r=0,82)$ et entre les échantillons, le rapport de ces acides varie du simple au double. L'épaisseur moyenne des échantillons de chaque lieu de prélèvement est significativement corrélée $(P<1 \%)$ avec la teneur en lipides $(r=-0,82)$ et le pourcentage des acides gras $(r=+0,75,+0,86$ et $-0,89$ respectivement pour $16: 0,18: 0$ et $18: 1, n-9)$. Ainsi, ces résultats montrent que la composition du tissu OM varie selon le lieu de prélèvement et, bien que la taille des cellules n'ait pas été mesurée, il semble que l'indice de saturation plus élevé dans les zones de dépôts puisse être lié au degré de remplissage des cellules et donc à l'état d'engraissement des animaux. La LPL, par contre, est plus active dans les échantillons situés près de l'attache du tissu OM sur le rumen et non sur l'ensemble des zones périphériques comme le suggérait Chilliard (3). Le lieu de prélèvement du tissu adipeux doit donc être défini avec précision pour l'étude du métabolisme lipidique de ce tissu. L'origine de ces variations devrait être étudiée en tenant compte de la vascularisation et de la cellularité de l'échantillon et en période d'anabolisme intense. Malgré le faible nombre d'échantillons dans les tissus PR et MES, il semble que les différences inter-échantillons soient significativement plus faibles dans le tissu PR que dans le tissu OM pour les teneurs en lipides $(P<10 \%)$, les activités $L P L(P<1 \%)$ et les pourcentages de $16: 0,18: 0$ et $18: 1, n-9(P<1 \%)$. Dans le tissu MES, en dehors de la LPL $(P<1 \%)$, ces différences sont du même ordre que dans le tissu OM. Ainsi, du point de vue de sa composition le tissu OM est le plus hétérogène et le tissu PR le plus homogène. Le tissu MES, le moins riche en lipides, présente l'activité LPL la plus forte tandis que le tissu $O M$ est le plus insaturé. Ces variations intra-tissu de ces critères ne masquent cependant pas les variations entre les tissus et entre les chèvres.

TABL. 1. - Composition et activité $L P L$ des tissus adipeux abdominaux, omental (OM), périrénal (PR), mésentérique (MES) chez la chèvre en fin de gestation.

\begin{tabular}{|c|c|c|c|c|c|c|c|c|c|}
\hline & \multicolumn{3}{|c|}{ Tissus adipeux } & \multicolumn{6}{|c|}{ Sources de variations (s) } \\
\hline & $\underset{x}{O M}$ & $\begin{array}{l}P R \\
x\end{array}$ & $\begin{array}{c}\text { MES } \\
x\end{array}$ & Chèvre & Tissu & $\begin{array}{c}\text { Sites } \\
\text { dans } \\
\text { OM }\end{array}$ & $\begin{array}{c}\text { Sites } \\
\text { dans } \\
\text { PR }\end{array}$ & $\begin{array}{l}\text { Sites } \\
\text { dans } \\
\text { MES }\end{array}$ & $\stackrel{\mathbf{s}}{\text { résiduel }}$ \\
\hline $\begin{array}{l}\text { Lipides } \\
\text { LPL } \\
16: 0(\%) \\
18: 0(\%) \\
18: 1, n-9(\%)\end{array}$ & $\begin{array}{l}92,9 \\
22,2 \\
29,9 \\
27,6 \\
25,6\end{array}$ & $\begin{array}{l}93,9 \\
21,8 \\
32,1 \\
30,7 \\
19,6\end{array}$ & $\begin{array}{l}89,1 \\
32,6 \\
29,0 \\
32,6 \\
20,7\end{array}$ & $\begin{array}{r}5,7^{* *} \\
43,4^{* *} \\
10,1^{* *} \\
8,4^{* *} \\
11,7^{* *}\end{array}$ & $\begin{array}{r}10,3^{* *} \\
25,9^{* *} \\
6,3^{* *} \\
13,1^{* *} \\
17,1^{* *}\end{array}$ & $\begin{array}{c}3,0^{*} \\
18,1^{*} \\
1,9^{*} \\
5,1^{* *} \\
5,6^{* *}\end{array}$ & $\begin{array}{l}1,8 \\
5,0 \\
0,7 \\
0,4 \\
1,7\end{array}$ & $\begin{array}{l}2,9 \\
2,9 \\
1,5 \\
4,9^{*} \\
4,7\end{array}$ & $\begin{array}{r}2,1 \\
12,7 \\
2,0 \\
2,4 \\
3,2\end{array}$ \\
\hline
\end{tabular}

Lipides : \% / tissu frais : LPL : lipoprotéine lipase $\mathrm{nm} / \mathrm{mn} / \mathrm{g}$ de lipides ; $\mathrm{x}:$ moyenne ; s : écart-type des effets ; ${ }^{*},{ }^{*}$ : effet significatif au seuil de $5 \%, 1 \%$

Anderson D. B., Kauffman R. G., Kastenschmidt L. L., 1972. J. Lipid Res., 13, 593-599. Bas P., Rouzeau A., Morand-Fehr P., 1984. Cah. Techn. I.N.R.A., 7, 43-45.

Chilliard Y., 1985. Thèse Doctorat ès Sciences Paris VI, $134 \mathrm{p}$.

Chilliard Y., Dorléans M., Fehr P. M., 1977. Ann. Biol. anim. Biophys., 17, 107-122.

Leat W. M. F., 1975. J. agric. Sci., Camb., 85, 551-558. 